\title{
Using methods of the reverse engineering to carry personalised preoperative stabilisers out on the example of vertebrae of human spine
}

\author{
Ewelina Kozłowska ${ }^{1, *}$, and Jarosław Zubrzycki ${ }^{1}$ \\ ${ }^{1}$ Lublin University of Technology, Faculty of Mechanical Engineering, Institute of Technological Systems of Information, Nadbystrzycka 38A, \\ 20-618 Lublin, Poland
}

\begin{abstract}
There is presented the possibility of using modern rapid prototyping methods in the form of reverse engineering on the example of lumbosacral spine of a human spine at the level of L3-L. Reverse engineering techniques were directly used in the generation and processing of point clouds from a real object. The point clouds were generated by using specialised Mimics software and basing on the results of computer tomography and magnetic resonance imaging using. In the next stage of processing, there was the export of the appropriately converted point clouds to STL format, compatible with CAD programs. The reverse engineering process took four steps: digitisation of the real object, using non-invasive imaging techniques (CT, MRI), processing the point clouds from the digitisation stage, construction of a surface model of the real object basing on the processed point clouds, realising of the real object's copy by using rapid prototyping techniques. In the final stage, the model of the complex spinal's part was tested to MES strength analysis.
\end{abstract}

\section{Introduction}

Nowadays, diseases and injures of a spine are included to illnesses from the group called: civilisation diseases. Thanks to the technological progress in the field of providing medical services, treating diseases and back injuries, there was a significant quality improvement in services dedicated to people from the risk group, as well as for people after different kind of accidents.

Long-term examinations contributed to the more effective development of non-invasive methods of the medical imaging, such as computed tomography (CT) and magnetic resonance (MRI), which acquiring a lot of more accurate data about anatomical structure of the entire human organism, particularly spinal's areas (entire area or partial).

Development of new technology in medicine, such as reverse engineering $[4,12,13]$ and artificial intelligence [1], enables to extend the chances in the field of the treatment of illnesses and damages of a spine. In the work, there was submitted to consideration directly concerning the most profitable choosing of treating definite diseases and damages of a spine on lumbarcross-shaped sections at the level L3-L5.

Currently, thanks to the intensive developing techniques of implantology, the spondylosis is increasingly becoming redundant because of its invasive [13-15]. The spondylosis is a surgical procedure of anastomosis neighbouring vertebrae with the assistance of specialist implants, which are needed to accelerate the process of osseous concretion with the simultaneous stabilisation of vertebrae in a correct way in the area of damage.

However, it is not always possible to use the treatment method for this type of illnesses by implantation an imitating degenerated implant or the degraded vertebra called artificial disk.

There is the possibility of developing directly, minimally invasive but efficient solution for a patient, in medical and technical aspect, which enables to direct support in treating process and further rehabilitation of a patient.

Applying the reverse engineering in the imitative process of the vertebra requires gaining and analysis of clinic necessary data describing the real state of the vertebra and the constitution of the patient. A database of possible solutions is being created in the area of curing diseases and injuries of the spine. Applying methods of the rapid prototyping enables to design and to produce personalised endoprosthesis, in the form of the implant directly reflecting the injured vertebra $[5,10]$. As a result, doctors receive above action data enabling them to make a rational decision in relation to the considered case, requiring the treatment at an outpatients' clinic on the neurosurgical and orthopaedic branch.

The direct source of ambulatory data is a process of analysis and aggregating of illnesses and traumas of backbone on the cross for small of back segment of patients, and results of non-invasive methods of diagnostics of medical (medical of imaging) image [9] presenting physiology and pathological changes that take place in the organism of patient.

Corresponding author: ewelina.kozlowska16@wp.pl 
Presently, the techniques of medical image used in medicine due to the widely developed possibilities which allow to execute researches considerably perfecting the process of medical diagnostics also help a simulation and preparation of operating procedures [10].

The main applied methods during non-invasive diagnostic of the medical imaging are examinations making with the help of the computed tomography (CT) and of magnetic resonance (MRI) [10]. In the article there is described process of the three-dimensional modelling situated in the lumbosacral part of spine at the level L3 - L5. In the next stage results coming directly from CT examinations and MRI made without applying the contrast will be used in the process of designing personalized endoprosthesis in the form of the implant reflecting the degenerate vertebra. The injured vertebra must be replaced to remove disease or the back injury from the patient at least invasive way. Afterwards there will be brought closer the process drawing the expert program up (advisory) basing directly on a clinic data included in the card of stories of the illness of the patient as well as on latest results of examinations coming from the computed tomography and the magnetic resonance.

\section{Anatomy and generalised functions of human spine}

The spine of the man is a column directly providing the support for the whole body. It directly consists of vertebrae and their connections. In its construction there are singled out the following elements:

1. 33-34 vertebrae,

2. 23 intervertebral disks (of so-called disks),

3 . the spinal cord along with spinal nerves,

4. cords $[8,13]$.

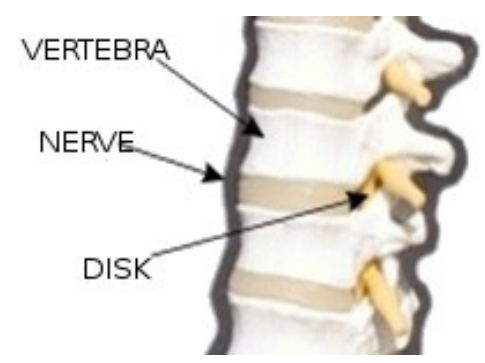

Fig. 1. Structure of the spine [8].

In the spinal construction five segments can be separated:

- cervical segment - 7 vertebrae keeping the head (C1 C7),

- deep segment - 12 vertebrae holding ribs (Th1 - Th12),

- the lumbar region - 7 vertebrae building the back wall of the belly (L1 - L5),

- the sacrum - healing over of 5 vertebrae (connection with the pelvis) - S1,

- coccyx - 3-4 integrated vertebrae (Fig. 2) [8, 9].

Generalised functions of human spine:

- holding the weight of the body and ensuring the balance,
- ensuring the fastening of other bones and elements of the human body,

- amortisation of shocks,

- protection of the spinal cord and spinal nerves.

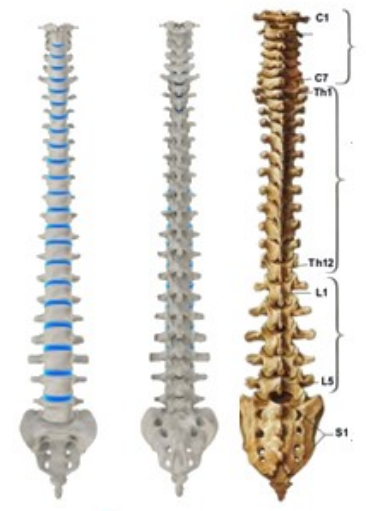

Fig. 2. Structure of the spine [17].

During the biological analysis of the structure of the lumbar vertebrae there is possibility to observe distinct flat spinous processes horizontally, placed towards the back, enabling to make moves of bending over towards the back of the lumbar region. Simultaneously, in the same part of the spine there are situated strong spinous crosswise, growing on the border of the hard core and the bow because of growing together residual lumbar ribs with the proper transverse process. The analysed lumbar region is deprived of all holes in transverse processes and the area joints for ribs.

Conducting anatomical analysis of the sacral vertebrae, it is possible to observe upper and bottom indents of the integrated sacral vertebrae, creating four pairs of sacral foramens joined directly to the vertebral canal. Sacral foramens are serving estuary to frontal branches, whereas towards the back branch of the dorsal cross nerves.

In the analysed lumbosacral part of the spine spinous processes (joint) are merging into the bone slat (median sacral crest), simultaneously integrated joint processes form two side slats (indirect cross backs). However crosswise processes are knitting into two lofty side sacral crests, and residual cross ribs form side parts of the sacrum. Owing to such a structure, vertebral canal is concealed directly under the centre comb and ends with the bottom hole, by which the filum terminale of the spinal cord is originating. Four appearing joint areas are creating the cross section: upper (merging with the last lumbar vertebrae) and two side (merging with bones of the pelvis at the lower ending) and caudal tangent with the coccygeal bone [6].

In the human spine, divided ranges are intervertebral disks between themselves, and the integrity of the structure is stabilised with cords of the spine constituting the depreciation simultaneously. Thanks to such a structure of the spine the outcome of curves is coinciding with the division, forming the straight silhouette at the man.

The intervertebral disk is built from the annulus fibrosus (outside part) and nucleus pulposus (inner part). 
Correctly formed nucleus pulposus is built in approx. $80 \%$ of water and is subject to the laws of hydrodynamics. Simultaneously, it plays crucial role during flexible moves in the spine through the migration into the side opposite to the made movement through the spine, however while bending the spine ahead, nucleus Atherosclerotic nucleus is moving in back direction. Mainly the intervertebral disk is serving as the shock absorber of the entire spine of the man (Fig. 3).

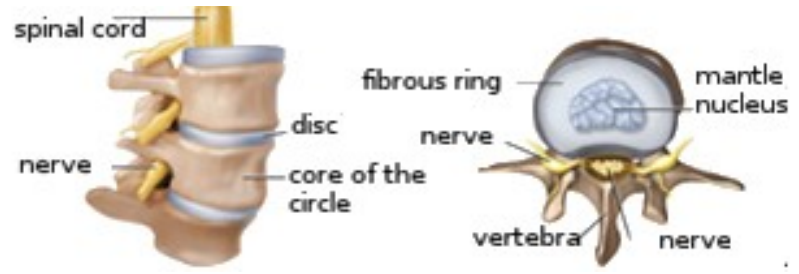

Fig. 3. Structure of the intervertebral disk $[3,16]$.

\section{Computer support of medical diagnostics imaging}

Today, the development of medical diagnostic techniques is directly based on computer-aided diagnosis (CADx) methods, which are widely used in medicine [11]. The usage of computer-aided diagnosis in medical imaging techniques (mammography, radiography, computer tomography, magnetic resonance imaging, ultrasonography) is the process of detection and evaluation of pathological changes. The wide spectrum of usability allows it to be used in clinical radiology, and in particular for the detection of spinal injuries and injuries in all its sections. In the concept of CADx, the radiologist attaches great importance to the prediction made by the computer, but the final decision belongs to the radiologist. By applying the CADx concept, the radiologist makes a good diagnosis, which, in case of doubt, can help with the suggestions of the computer [7, 15].

Computer image diagnostics support is intended to enhance the human ability to solve key problems of image interpretation. Upgrades and improvements to imaging techniques and alleviation detection and differential diagnosis algorithms are possible thanks to the ongoing research on CADx algorithms. Currently, CADx technologies are implemented directly into the PACS system, followed by the creation of collections of pathologies of similar nature.

Dynamic technological development and simultaneous improvement of the functionality of computer software and its use in digital imaging enable the use of CADx in most diagnostic methods [8]. However, the way to use CADx depends directly on the doctor's preference (radiologist), who can make a final diagnosis and help with the suggestion of the program. Unfortunately, the above procedure has a negative side, which is a waste of time devoted to conducting the study and issuing the opinion. In the above case, it is better to perform a preliminary analysis of the image by the computer and then subject it to a secondary evaluation by a doctor. Despite the extensive capabilities of the computer, even in this case there is a risk of missing some significant damage or deformation not captured by the computer $[1,6]$.

\section{Design 3D cross for small of back segment at the level L3-L5}

3D modelling process of the lumbosacral segment at the level L3 - L5 was made based on tomographic findings and after the stage of the process of the digitisation [12] (Fig. 4).

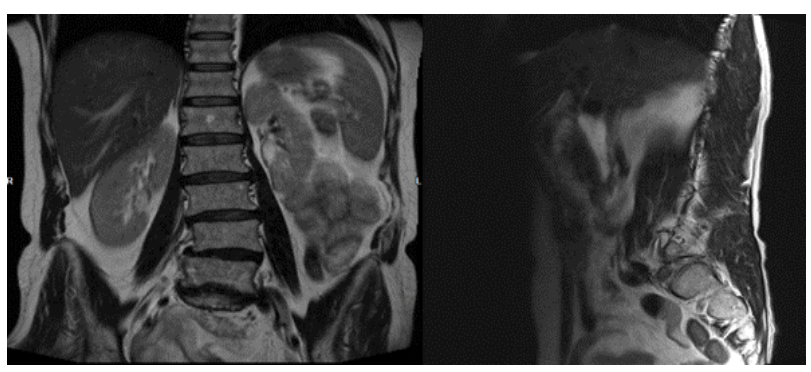

Fig. 4. DICOM image of the lumbar-sacral segment.

Basing on DICOM 2D obtained from CT and MRI using the Materialise Interactive Medical Image Control System images, there was generated the $3 \mathrm{D}$ model of the spine in a particular section. The above program is a specialised software for directly processing medical images and creating 3D models, as well as the "3Matic" module for numerical analysis.

After loading a series of DICOM images into Mimics, the program displays the 2D image in three dimensions (Fig. 4, 5): frontal, axial, sagittal and 3D view. The axial view comes directly from the import of a series of images, while the frontal and sagittal views are generated automatically by the program. In Mimics, all views are associative, i.e. each time you click on the image with the left mouse button, it automatically updates the other views [2, 11] (Fig. 5, 6).
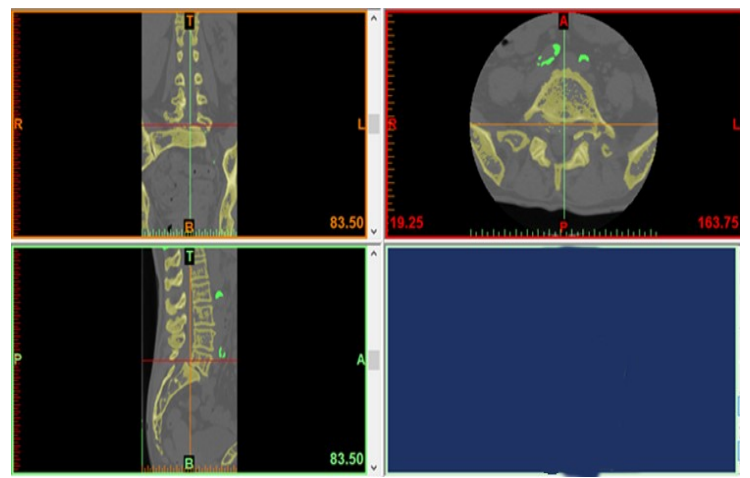

Fig. 5. View of the spine in the Mimics program after loading series of 2D photographs.

Then, after loading the DICOM files and transforming using the main tools like "Thresholding" (selection of tissue type using the HU range), "Growing Area" (selection and interpolation of areas that meet the criteria of the previous operation) and "Calculate 3D" The process of generating a $3 \mathrm{D}$ skeleton model of the spine as a digital model of a real object. The obtained 3D 
model is a numerical reflection of the real dimensions of the spine of a given patient. Thanks to the above operations there is the possibility to generate a model which is adapted individually to the anatomical dimensions of a particular patient. The final effect of modelling is shown in Fig. 7.
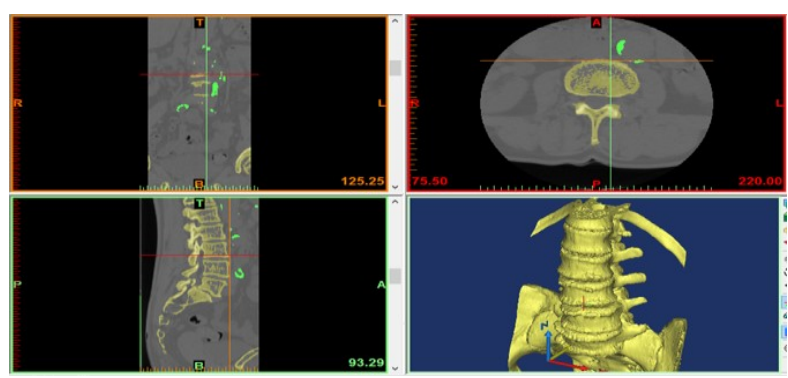

Fig. 6. View after loading series of photographs with the generated model 3D.

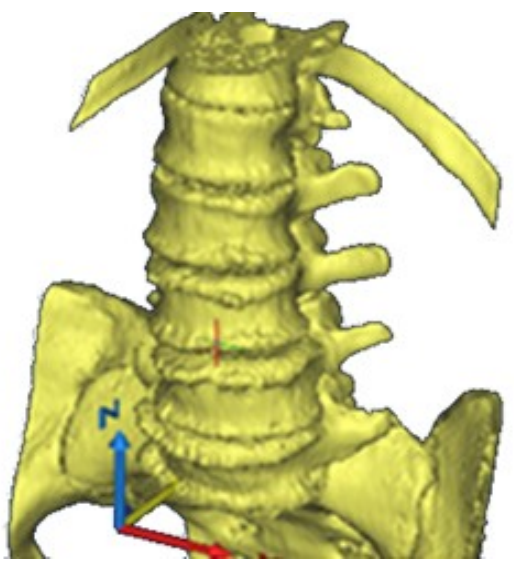

Fig. 7. Model 3D recreated section with the visible discontinuities of surface.

Visible 3D model of surface discontinuities area natural phenomenon occurring during model generation. They result are directly from the quality of data in DICOM format. However, with the "Edit Mask-Draw" function, it is possible to remove small deformation model imperfections, which the further stage could lead to incorrectly generating a correct STL file.

The next step in 3D lumbar-area modelling is the process of reconstructing its geometry by exporting the generated STL file to CAD programs for further processing and design of the surface model and CAE programs for numerical calculation and numerical simulation. Despite the many practical features of the Mimics program to further analysis the model which was generated in a above program there is a necessity of export to the Geomagic Design X. In the first stage, the Geomagic Design $X$ program removes the generated discontinuities and distortions. Due to the presence of multiple discontinuities in $3 \mathrm{D}$, it is not possible to generate a valid and complete STL file. In order to improve the quality of the file, there was used the feature for save the "Point Cloud" which enables to save data directly into point clouds which are not dependent on each other and described coordinates.

Further stages of L3-L5 lumbar-sacral spine modelling and reconstruction were generated in the
Geomagic Design X program. The Geomagic Design X system provides a module for $3 \mathrm{D}$ design using digitized data. The "Digitized Shape Editor" is located in the shape design module "Shape". The above command is used directly to process data in the form of point clouds, triangular or quadrilateral networks.

Through the import of the STL file generated in the Mimics program next step is loading it as the cloud points which will be subjected to reconstruction is taking place. In a dialog box in part "Preview", after the keystroke "Update", a visualisation of the imported cloud points and activating are taking place of automatic creating the surface (Fig. 8).

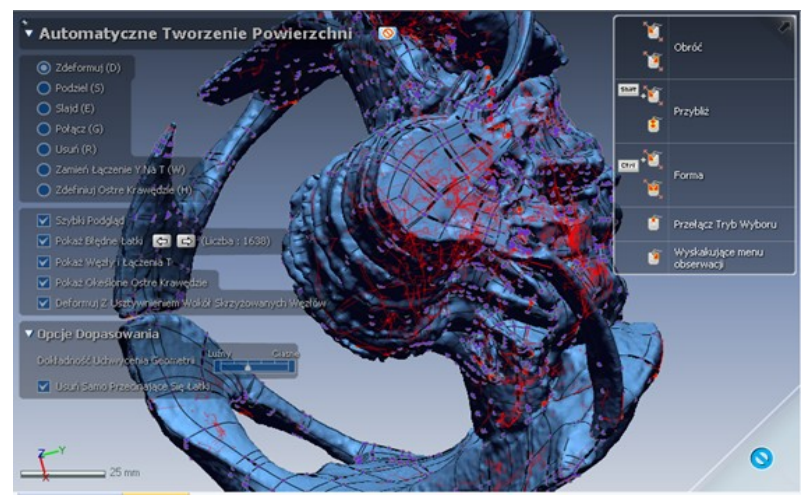

Fig. 8. Loading cloud points to the Geomagic Design Program X.

In Fig. 8 a model of the cloud points depicting the entity object, having elements in their outline incorrectly defined and classified creating the cloud of points and making it difficult for the area to create is visible on the model (red points).

In the further step generating the spot model in the form of networks of curves circumscribing established outlines of vertebrae is taking place what enables the visualisation of the shape of the skeleton of spine 2D (Fig. 9).

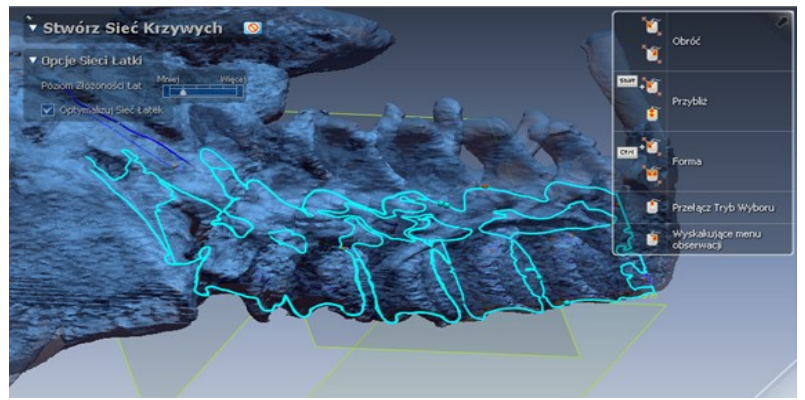

Fig. 9. Creating Curve Networks in Geomagic Design X.

After realising the process of generating 3D from the cloud points of $3 \mathrm{D}$ model containing only one L3 vertebra, a created surface model is essential to conduct the numerical analysis in CAE programs. The STL file generated correctly in the Geomagic Design Program X enables the NX 11.0 CAD/CAM to create the surface model in the program from the group, with STL which has got extended orders and modules enabling the export and direct processing of files (Fig. 10). 


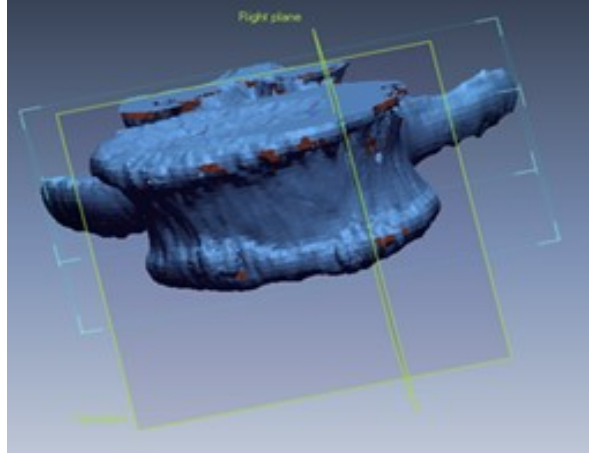

Fig. 10. 3D model of L3 vertebra with visible surface discontinuities.

In the next stage of the modelling of the cloud basing on correctly generated cloud points there is creation of the grid squares situated on points of the virtual surface allowing to draw the surface model (Fig. 11).

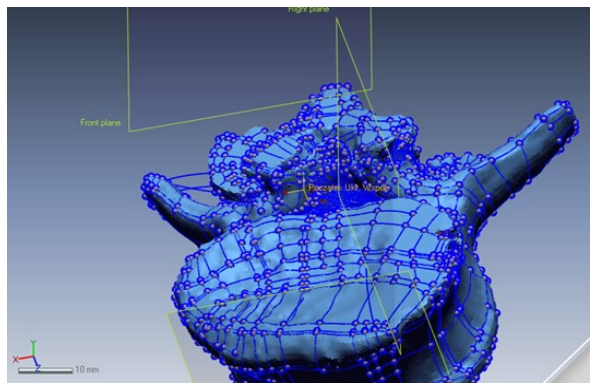

Fig. 11. 3D model of L3 vertebra with visible nodes on a grid of polygons.

The working out of a correct model of a geometric recreated object occurs through the processing of cloud points obtained directly from the process of digitalisation and because of the removal of all points of measuring unconnected with recreated the object (filtration of sounds, smoothing, filling losses in the structure of surface).

Therefore, the processed measuring cloud points grows into a spatial object that consists of quadrangles. The projected model is subjected to further treatment, through repairing multiparty nets (polishing, implementation of losses, cleating of different groups of polygons, treatment of border or sharpening of corners). Those operations aim to prepare appropriate data that allow realising the last phase of model creation of the real geometric as shreds of surface.

The designed surface model in the next stage was converted into the homogeneous solid. For that purpose, they used the function "Join" and "Close Surface" from the module "Reverse Engineering" (Fig. 12).
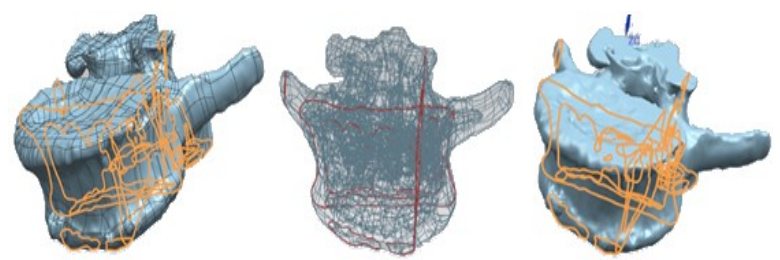

Fig. 12. Surface model of the L3 vertebra with visible crosssections.
After the realisation of all stages of reconstructing of the real model and the rendering of virtual model 3D a solid model of the L3 vertebra came into existence (Fig. 13).

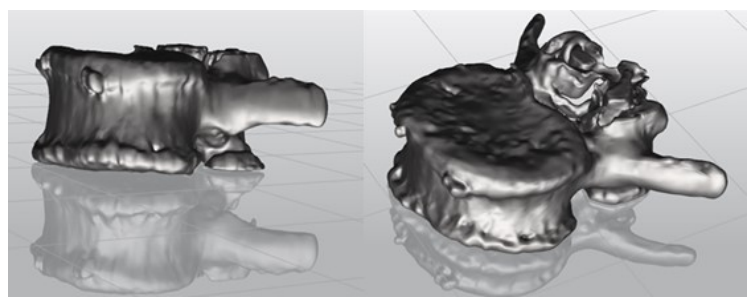

Fig. 13. The solid model of the L3 vertebra was formed from the transformation of the surface model.

The designed solid model allows analysis of the type and size of the deformation of the vertebra in the NX NASTRAN module in NX 11.0 (Fig. 14).
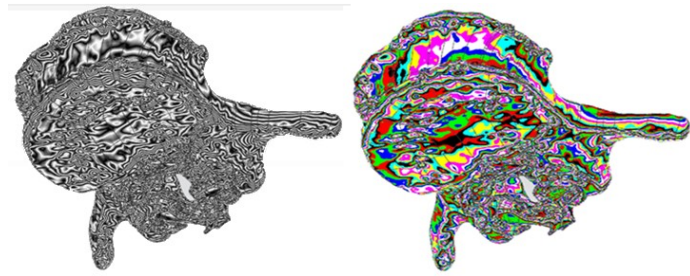

Fig. 14. 3D model of the L3 vertebra with visible deformations and structural defects.

Analysis of the type and size of the deformation shows that the greatest damage to the vertebrae of the vertebral column occurs at the site of the direct adhesion of the intervertebral disc to the vertebrae. The above damages result from both the errors generated during model generation from CT and MRI images and actual damage caused by a disease or spinal injury.

Visible significant deformations on the model in places of planting the spinal cord, of dorsal root of the spinal cord and on the body of vertebra surcharges of the checked length of the lumbosacral spine are effects at the level L3-L5 and strong emphases triggered by transferring the intervertebral disk surrounding the above disease section.

\section{Numerical analysis of the L3 vertebra in the CAE program}

A L3 vertebra model of the lumbar-sacral spine of the human spine was developed in Abaqus program. A strength analysis was realised in the case of a pressure load on the upper surface of the modelled L3 vertebra. This load allowed us to directly determine the state of stresses in each region of the disc. The isotropic material Ti-6A1-4V material was used for numerical analysis: Young's modulus: $\mathrm{E}=1.74 \mathrm{e}+04 \mathrm{MPa}$ and Poisson's number: $v=0.44$.

Static analysis was conducted directly on the designed L3 vertebra surface area model to obtain the regions which are exposed to damages - the largest concentrations of stresses according to the Huber-Misses hypothesis. According to the energy hypothesis of strain 
deformation, the form of strain is the energy of the strain which must satisfy the strength condition [18]:

$$
\sigma_{\mathrm{red}}=\frac{\sqrt{2}}{2} \sqrt{\left(\sigma_{1}-\sigma_{2}\right)^{2}+\left(\sigma_{2}-\sigma_{3}\right)^{2}+\left(\sigma_{3}-\sigma_{1}\right)^{2}} \leq k_{\gamma}
$$

Above regions were encumbered with the pressure $\mathrm{p}=190000 \mathrm{MPa}$, acting evenly on an upper surface of the L3 body of vertebra (Fig. 15).
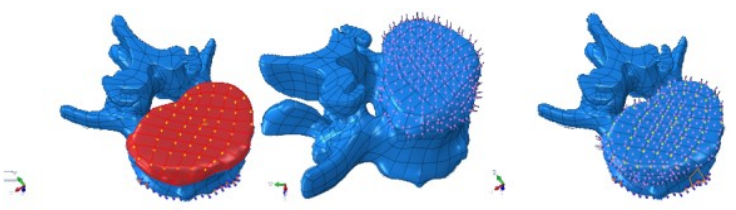

Fig. 15. Model vertebra L3 with the visible load.

And so, on the lower area of the analysed vertebra the numerical model was placed getting back all degrees of freedom. (Fig. 16).

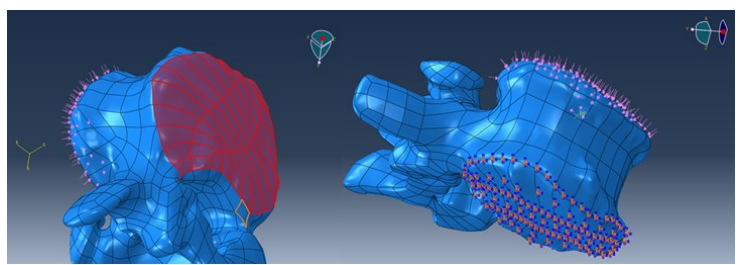

Fig. 16. Model vertebra L3 with visible placing.

The analysis allowed us to directly appoint the stress states of the Huber-Misses hypothesis and the value of the deformations caused by the pressure effect of the set value in the $\mathrm{L} 3$ region.

\section{Results and discussion of the numerical analysis of the L3 vertebra}

The numerical analysis of the state of overloading in the form of the pressure was performed for the physiological L3 vertebra of the lumbosacral spine segment. The upper surface of the L3 vertebra has been overloaded evenly.

The analysis of results, achieved from calculations done basing on assumptions of the hypothesis HuberaMisesa, indicates on the biggest concentration of reduced stresses in regions adjoining directly to the isthmus of the L3 vertebral (Fig. 17).

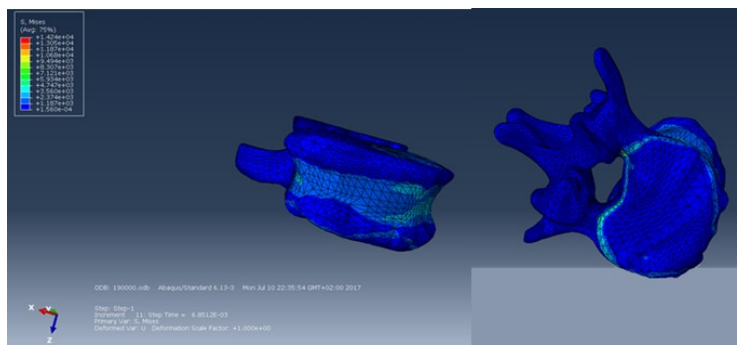

Fig. 17. Model vertebra L3 with visible deformations.

In case of the functioning of vertebras in real conditions these places are the most overloaded and exposed to coming into existence of microfractures, caused by longer working hours in the appearance of damage of the vertebra and the intervertebral disk.

With the inflicted value of load by the pressure $\mathrm{p}=190000 \mathrm{MPa}$ reduced strains were shaped on the level $-1,560 \mathrm{e}-04$ and 1,424e+04 MPa.

There were observed the minimal translocation of an upper surface causing dent of a central surface vertebra L3 giving $-2,608 \mathrm{e}+04$ and $8,434 \mathrm{e}+03$, which occurred the point accumulations of the tensions in vertebra L3 (Fig. 18).

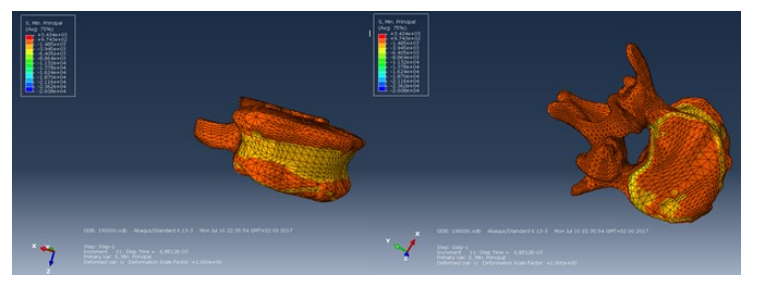

Fig. 18. Model vertebra L3 with visible displacement.

The maximum deformations caused by the effect of the pressure appeared directly in regions of spot masses of stresses at the level $-1.008 \mathrm{e}+04$ and $1.513 \mathrm{e}+04$ $\mathrm{MPa}$ (Fig. 19).

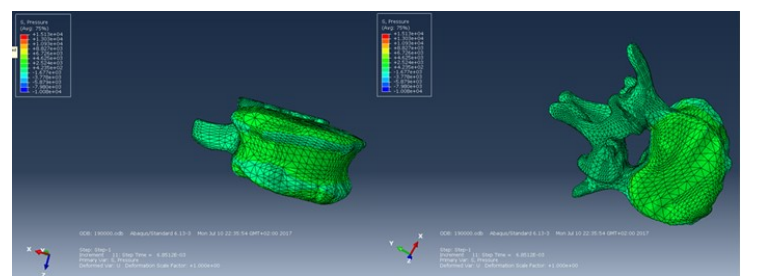

Fig. 19. Model of vertebra L3 with visible deformations caused by pressure.

\section{Conclusion}

The paper presents the possibilities of lumbar spine reconstruction technique at the level L3-L5. The reconstruction process was conducted basing on serial photos taken by computer tomography and magnetic resonance imaging.

The basic element of modelling process of the model (spine vertebra) was $2 \mathrm{D}$ serial photos of the backbone at the level L3-L5 recorded during CT and magnetic resonance imaging, and directly recorded in DICOM format.

The computerised engineering support system enables to direct design of a virtual vertebral column model, showing the doctor spatial representation of the structures visible in CT images as cross-sections. The designed virtual model can be used in the surgical planning process and, at the same time, Intraoperative measurement is one of the methods of rapid prototyping.

Technique for the design and deformation of bone tissue directly forming the vertebral body is also used for planning and carrying out surgical procedures consisting in filling cavities in a vertebral structure with a specially prepared cement structure. Basing on DICOM images obtained by computer imaging methods, pathological areas of the vertebrae may undergo a reconstruction process in the CAD system. Thanks to the wide design 
capabilities of CAD systems a damaged vertebra design is created which is reconstructed in the next stage by direct removal of deformation and distortion of the vertebral body. After designing both models, they are overlapped and pointed where there are defects and discontinuities to inject the specially prepared agent. However, there are also cases in which the damage is already over-developed, and the usage of cement will not produce the desired effect. In this case, another implant treatment or stabilisation treatment is used.

The analysed 3D model of vertebra L3 lumbosacral section of human's spine was generated in the shape of cloud point at the beginning of the process by Mimics Materialise program, afterwards the model was exported to STL format and loaded into Geomagic Design X program, then in the next stage exported into $\mathrm{CAD} / \mathrm{CAE}$ NX 11.0. In the paper, there was presented the scheme of actions to be done in sequence to obtain correct 3D model which can be used to conducting numerical analysis in program for strength calculation. In further parts of the work there were done calculations of strength and static deformations. Those calculations do not take into consideration vertebra cracking. Despite avoiding vertebra cracking, there is a possibility to predict damage level.

The intensive development of multidisciplinary techniques and medicine fields enables to decrease time for modelling, making an implant or stabiliser significantly and decreasing treating and rehabilitation time too.

The calculations above do not consider the fracture (fracture) of the vertebrae. Despite the absence of cracking, the extent of damage and disease progression can be determined directly based on the fracture.

The paper presents a direct influence on the physiological load on the lumbar-sacral spine segment on the functionality and viability of the L3 vertebrae. Numerical analyses of static MES enabled to definite interdependencies between the physical state of the tissues, the mechanical load and the potential for lesions in certain regions of vertebra L3.

In the later stages of the work, the model will be developed by modelling L3-L5 lumbar spine vertebrae by modelling the entire vertebrae of a defined angle of lordosis. The above modelling and numerical analyses will be aimed at broadening the knowledge of biomechanical phenomena occurring directly in the system (L3-L5 spine model) where the implant is implanted in place of the damaged vertebra as well as in the system with spine stabilisation applied in the lumbarsacral stretch in the states of natural functioning as well as stress states.

\section{References}

1. E. Eilam, Reversing: Secrets of reverse engineering (John Wiley \& Sons, 2011)

2. T. Várady, R. R. Martin, J. Cox, Comput. Des. 29, 255-268 (1997)

3. J. Zubrzycki, M. Braniewska, Mechanik. 90, 46-47 (2017)

4. J. Barrat, Our final invention: Artificial intelligence and the end of the human era (Macmillan, 2013)

5. J. Zubrzycki, N. Smidova, in Applied Mechanics and Materials 613, pp. 172-181(2014)

6. T. Grimm, User's guide to rapid prototyping (Society of Manufacturing Engineers, 2004)

7. J. Tritt-Goc, Tomografia magnetyczno-rezonansowa, Foton 96, 157-165 (2007)

8. R. Putz, R. Pabst., Atlas anatomii czlowieka-Sobotta (Elsevier Urban \& Partner, 2001)

9. M. Mańko, J. P. Zubrzycki, R. Karpiński, J. Technol. Exploit. Mech. Eng. 1, 33-57 (2015)

10. R. Karpiński, Ł. Jaworski, J. Zubrzycki, others, Adv. Sci. Technol. Res. J. 10, 240-246 (2016).

11. A. Bochenek A., M. Reicher, Anatomia człowieka (Wydawnictwo Lekarskie PZWL, 1998)

12. L. Tsochatzidis, K. Zagoris, N. Arikidis, A. Karahaliou, L. Costaridou, I. Pratikakis, Pattern Recognit. 71, 106-117 (2017)

13. K. Kiszka, J. Haduch, M. Pasowicz, Przegląd lekarski 66, 437-447 (2009)

14. S. B Bartkowski, Chirurgia szczękowo-twarzowa (Collegium Medicum UJ, 1996)

15. http://drkregoslup.pl/leczenie-chirurgicznestabilizacja-ledzwiowa/leczeniechirurgiczne/stabilizacja-kregoslupa (08-2017)

16. https:/www.twojkregoslup.pl/kregoslup/budowa-ifunkcje-ogolne-kregoslupa (08-2017) 